\title{
A Hybrid Approach for Antenna Optimization Using Cat Swarm based Genetic Optimization
}

\author{
A. Singh ${ }^{* 1}$, R. M. Mehra ${ }^{2}$, V. K. Pandey ${ }^{3}$ \\ ${ }^{* 1}$ Department of ECE, Sharda University, Greater Noida, India-201306 \\ ${ }^{2}$ Department of ECE, Sharda University, Greater Noida, India-201306 \\ ${ }^{3}$ Department of ECE, Noida Institute of Engineering and Technology, Greater Noida, India-201306 \\ * corresponding author, E-mail: anushman.phd@gmail.com
}

\begin{abstract}
The aim of the paper is to introduce the hybrid technique for the multi objective optimization of antennas. The goal of the antenna optimization is typically minimising the reflection coefficient through a frequency band. To minimize the energy consumption is essential consideration of energy efficient transmission schemes that is used for the data transfer in wireless sensor networks. In our proposed work the efficient and low-cost multi objective technique CSGO (Cat Swarm based Genetic optimization) approach was used. The Cat Swarm Optimization approach is combined with genetic algorithm (GA) to optimize the bandwidth and return loss of the antenna. CSGO approach is to improve the optimization efficiency and simulation complexity. This hybrid optimization approach will reduce the side lobe level and provide improvement in the Directivity. CSGO is applied to the design of a miniaturized multiband antenna, showing better diversity and significant savings of overall optimization cost compared with the previously reported design methods.
\end{abstract}

Keywords: Cat Swarm based Genetic optimization, multi objective optimization, antenna structures, genetic algorithm, micro strip patch antenna, directivity, Cat swarm optimization, energy efficient, hybrid approach.

\section{Introduction}

Antennas play a very important role in the field of wireless communications. Antenna is a vital part of any electronic system which transmits or receives electromagnetic energy in a wireless mode. In most antenna optimization problems, several goals should be satisfied concurrently in order to obtain an optimal solution. The primary design objective of the antenna optimization is typically minimising the reflection coefficient through a frequency band. Microstrip Patch antenna (MPA) provides low outline and low
quantity[1].The developments of wireless communication industry continue to derive necessity of small, compatible and affordable microstrip patch antennas [2].Antenna is the most basic part in wireless communication systems. Antenna transmits the electrical signals into radio waves .The antennas characteristics depends on the need of signal transmission and reception [3]. microstrip antenna shapes such as square, rectangular, circular and elliptical, but any unremitting shape is feasible. The rectangular patch antenna was commonly used.

Microstrip antennas mostly used the microwave frequencies it is directly tied to the wavelength frequency. Antenna optimization is to create the advanced complex electromagnetic devices they determine conditions of performance, serviceability and cost [4]. Microstrip antennas had low weight, fabrication cost and mechanically in frequency operation. These antennas are used in many communications systems like as personal communication systems, satellite and other wireless applications [5]. Microstrip patch antennas are used in vital part of communication because they can be printed directly onto a circuit board. The antennas are becoming very wide in the mobile phone market . Patch antennas are low cost, low profile \& easily fabricated. These are relatively inexpensive to manufacture \& design because of the simple 2dimensional physical geometry. These are also light weight, conformal shaped, capable of dual \& triple frequency operations [6]. To improve global optimization capability for multi-objective design of multi-parameter antenna structures many techniques have been used to attempt to achieve this reduction in size with varying success. It has been shown that increasing the effective dielectric constant of the substrate will lead to decreased dimensions.

The genetic algorithm (GA) has the prospective of allowing the computer to design the antenna without an initial model. Genetic algorithms are probabilistic 
optimization processes that start from a population of randomly-generated potential solutions, and gradually evolve toward better solutions through a repetitive application of genetic operators. There are many antenna classes, such as dish antennas, phased array antennas (consisting of multiple regularly spaced elements), wire antennas, horn antennas, and microstrip and patch antennas. Each of these classes uses different structures and exploits different properties of electromagnetic waves. Wire antennas will be the focus of this paper. An antenna is a wire antenna if it is constructed from conductors that are much longer than their width [7].

A ground plane can reduce the required height and construction of the wire antenna [8]. Directivity and gain are two related qualities in antenna design. Directivity is the ratio of power density being transmitted by an antenna in a particular direction to the average power density being transmitted in all directions. The gain is the directivity multiplied by the ratio of power radiated to power input. Gain takes into account all losses such as loss due to resistance in the antenna, which converts some of the input power into heat, and loss due to mismatch between the transmitter/receiver and the antenna. Bandwidth is the useful range of frequencies for an antenna, and is usually desired to be as large as possible. It is given in percent, which is the ratio of the useful frequency span over the nominal operating frequency. The gain pattern or antenna pattern was plots gain magnitude versus angle; it shows the proportion of the antenna power. Polarization refers to the orientation of electromagnetic waves. Electromagnetic waves are composed of two components: an E-field (electric field) component, which is a sinusoidal wave that exists in one plane, and an $\mathrm{H}$-field (magnetic field) component that exists at right angles to the E-field [9].

Cat Swarm Optimization is combined with a genetic algorithm (GA) to optimize the bandwidth and return loss of the antenna. This hybrid optimization algorithm will reduce the side lobe level and provide improvement in the Directivity. Our approach is also applied to the design of a miniaturized multiband antenna, showing better diversity and significant savings of overall optimization cost compared with the previously reported design methods. The CSO have two sub-models they are seeking mode and the tracing mode. To merging this to algorithm, we define a mixture ratio (MR) of joining seeking mode together with tracing mode. Section 2 gives the some of the recent research work done in the antenna optimization technology .The proposed methodology and its explanation are given in section 3 . Section 4 gives the simulation results and the performance comparison of the proposed method with the other traditional methods and followed by conclusion which presents in section 5 .

\section{Related Works}

The very recent works related to the multi objective optimization in antenna is given as follows.

Darshana et al [10] had proposed the Design of Rectangular Microstrip Patch Antenna Using Particle Swarm Optimization. In this Particle swarm optimization (PSO) is a popular optimization algorithm used for the design of microstrip patch antenna. He was presented design using soft computing technique, PSO of probe fed rectangular microstrip patch antenna for WCDMA. For the design of microstrip patch antenna a substrate with dielectric constant of 4.4 and height $1.588 \mathrm{~mm}$ has been used. To optimize the parameters PSO has been used and parameters like patch length, width and feed position at center frequency of $1.95 \mathrm{GHz}$ using Sonnet13.52. Microstrip patch antenna resonated at exact $1.95 \mathrm{GHz}$. PSO saves time as compared to the design of patch antenna without optimization algorithm and also PSO restricts the variation from center frequency.

Al-Jodah et al [11] had introduced the method as Spider Monkey Optimization (SMO) for solving electromagnetic problems. The paper was to introduce the SMO method and to demonstrate it effectiveness to allow this algorithm to join other popular evolutionary optimization techniques as a useful tool for electromagnetic problems. The SMO method was used in the synthesis of linear array antenna for the purpose of suppressed sidelobes and null placement in certain directions. Moreover, the SMO had been linked to full 3D electromagnetic simulation software to find the optimal dimension of an E-shaped patch antenna. The performance comparison of the SMO algorithm with the other well-known techniques had been illustrated .

Poian $M$ et al [12]. had presented an energy efficient scheme of joint antenna-subcarrier-power allocation for min-rate guaranteed services in the downlink multiuser orthogonal frequency division multiplexing distributed antenna systems (OFDM-DASs) with limited backhaul capacity. To maximize the energy 
efficiency in an OFDM-DAS based on the constraints of users' Quality of Service (QoS), subcarrier reuse, backhaul capacity and remote antenna units' transmit power. This scheme achieves good performance on energy efficiency as well as spectral efficiency and energy consumption, which is important in designing energy-efficient wireless communication systems. The maximum transmit power is relatively low. Numerical results demonstrate the effectiveness of the proposed low-complexity energy-efficient scheme and illustrate the fundamental trade-off among energy consumption, spectral efficiency and energy efficiency.

Koziel et al [13]. had presented a low-cost multi objective optimization for antenna . The approach was exploration of the Pareto front representing the best possible trade-offs between conflicting objectives, here, the structure size and its electrical performance. Starting from the design representing the best in-band reflection level, subsequent Pareto-optimal designs were identified through local constrained optimization aimed at reducing the structure size while maintaining the prescribed thresholds concerning the reflection response. Design techniques exploiting surrogatebased optimization (SBO) have been proposed recently to alleviate this difficulty .

Slawomir et al [14]. had presented a technique for reliable EM-driven size reduction of antenna structures. That approach exploits relaxation of design objectives which allows for achieving smaller footprint area compared to conventional formulation of the design problem as demonstrated by examples. Numerical results have been validated experimentally. The techniques mentioned above utilized to increase geometrical complexity of the antenna and introduce additional parameters that have to be adjusted to satisfy given performance requirements. The relaxation algorithm alleviating the issue of limited number of geometry parameters directly affect the antenna size [15].

Kriti Priya and Gupta[16] had presented a Genetic Algorithm Approach for Bandwidth Optimization in Near Video on Demand System. All the schemes were compared on the basis of the required bandwidth i.e. average number of I/O streams. The optimal batching time and the minimum streams required were determined by using GA. Numerical results were provided for verifying the analytical results with the GA results. The problems of optimizing the resource bandwidth for the video server have been solved by using the conventional linear programming or nonlinear programming approaches depending on the nature of the objective function.

Lisboa et al [17]. presented a multi objective optimization to antenna design, emphasizing the main general steps in this process. Specifications of antennas usually involve many conflicting objectives related to directivity, impedance matching, crosspolarization, and frequency range. For problems with more than one objective, the concept of optimum becomes more complex, and it was qualified as Pareto optimum. In this concept, for instance, the set of parameters where the directivity is maximum was not necessarily where the standing-wave ratio is minimum, so that tradeoffs between them become also an optimum in the sense that no point in the Pareto set has both objectives better than another point [16].

Modi et al [18] had presented a Neural Network model for the design of an Elliptical Patch Micro-strip Antenna (EPMSA) for a desired frequency in L Band. The artificial neural network (ANN) has been trained for any desired gain at desired frequency in L-band it can generate design parameters of EPMSA with great accuracy. The ANNs include gain at resonant frequency for the synthesis of EPMSA and was easy to achieve accurate result. By this method various parameters required for the design of an EPMSA at a particular frequency and gain of interest was easily extracted without going into the rigorous time consuming, iterative design procedures using a costly software package

Pujara et al [19]. presented an adaptive neuro fuzzy inference system (ANFIS)-based model for predicting the performance of a pyramidal and a conical corrugated horn antenna. Using these models, horn return-loss characteristics and radiation patterns (copolarization and cross-polarization) was obtained with great accuracy for any combination of design parameters. In this method less time and minimum computational resources was acquired, then the return-loss and radiation characteristics of a horn antenna under test was obtained with high degree of accuracy.

\section{Fast Multi Objective Optimizations in Antennas}

Multi objective optimization involves more than one objective function, the task of finding one or more 
optimal solution. An antenna design usually involves specifications related to directivity, impedance matching, cross-polarization, and frequency range, which can be formulated as both objective and constraint functions. The optimization problem of antenna design is evaluating the computational cost which is efficient one. The Good designers can define meaningful optimization problems, first it reduce the search space, and provide good starting points [20]. There are three significant parameters for design of a microstrip Patch Antenna such as Frequency, Substrate thickness and dielectric substrate. The thick dielectric substrate have low dielectric constant is desirable, it provide a better efficiency, larger bandwidth and better radiation. The optimization can always be manually stopped if no further improvements are expected [21]. The cat swarm optimization technique to find estimate solutions to enormously impossible numeric maximization and minimization problems. The system is flexible because agents can be easily added or removed without influencing the structure. Antenna parameter is radiation pattern, directivity, radiation resistance and efficiency, power gain, beam width and directivity. Minor lobes usually represent the radiation in undesired directions, and minimized. Side lobes are biggest to the minor lobes. The minor lobes are usually expressed as a ratio of the power density. In the radar systems, low side lobe ratios are very important to minimize the false target indications through the side lobes (e.g. $-30 \mathrm{~dB}$ ). The beam width of an antenna is a very central figure of merit and often is used as a trade-off between it and the side lobe level, the beam width decreases, the side lobe increases. The ratio of the radiation intensity in a given direction from the antenna to the radiation intensity averaged over all directions.

The below figure. 1 shows the block diagram of CSGO optimization it is combined with Cat Swarm Optimization and Genetic optimization .The cat swarm optimization algorithm (CSO) is one of the evolutionary computation algorithm. CSO is a high performance computational method and it is reducing the side lobe level then improvement in the Directivity [22].CSO used for discrete and continuous optimization problems. CSO is applied to optimize the individual element positions to achieve minimum side lobe levels and also for placement of nulls in desired directions . Genetic algorithms are evolutionary algorithms that use the parameter like as inheritance, mutation, selection, and crossover. A typical GA requires the defined genetic representation and fitness function. The genetic representation is provide domain solution and fitness function is to evaluate the solution domain. The CSO approach is finding the best global solution and achieves the better accuracy level of convergence in less iteration.

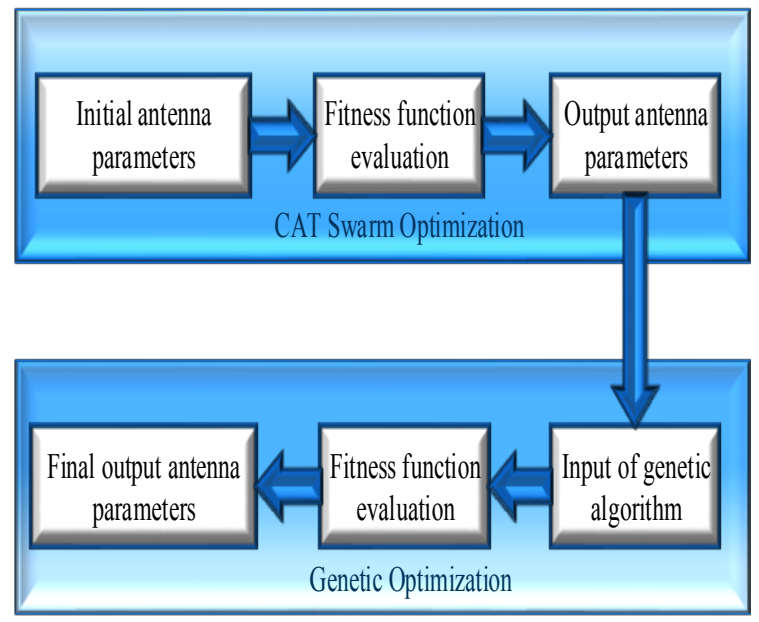

Figure 1: CSGO Block Diagram

We used the following optimization algorithms in antenna:

Genetic Algorithm (GE): To find the fitness value of each member of a random population and to choose the individuals with the best fitness value for further reproduction.

Particle Swarm Optimization (PSO): PSO approach is similar to GA. System initialization starts with a population of random solutions and searches for optimal by updating generations. This algorithm has no evolution operators such as crossover and mutation.

Cat Swarm Optimization (CSO): It has been improved and applied in different fields. CSO model analyze the behaviour of cats and they mostly except when they are tracking some targets.

Differential Evolution (DE): It was used to solve optimization problems where the objective function is non-differentiable, non-continuous, and non-linear. It uses D-dimensional parameter for a search for each generation.

Legendre Polynomials (LEG): This approach is also called Legendre functions. The particular orthogonal functions used for the solution of physical problems. The evolutionary algorithms usually had a better performance regarding sidelobe level reduction in antenna arrays.

\subsection{Antenna Efficiency}

Antenna efficiency and antenna illumination or aperture efficiency are less straightforward. An antenna is a device that is made to efficiently radiate and receive radiated electromagnetic waves. In two 
way communication the same antenna can be used for transmission and reception. Transmission means radiates electromagnetic energy into space and the reception part its collects electromagnetic energy from space. Antenna parameters bandwidth may be significantly reduced. Antenna synthesis methods accept the long computational time because evolutionary algorithm is often the only possible method to obtain a very high-quality design. But, a few works have been conducted concentrating on efficiency enhancement. They can be classified into the following categories. Space mapping is the main limitation of such methods is that the success of the space mapping mechanism comes from the basic assumption that the optimal points of the coarse and the fine models are not far away from each other in the design space. However, this is not always true in practical antenna design and often cannot be known beforehand. Cooperation with EM simulation is the speed of optimization can be substantially increased by only solving a single electromagnetic computation problem for the whole optimization process. The main idea of improving the search engine is able to obtain a suitable solution with a reduced number of exact function evaluations.

Antenna performance is evaluated during optimization as a linear combination of three parameters. These parameters are the Q-factor, the difference between the stored electric and magnetic energies, and metallic area. They have been chosen to illustrate the single frequency antenna Q computation method. Other important parameters such as losses, radiation resistance or matching are not considered here. The energy-difference mentioned above represents the quantitative measure of self-resonance used during optimization.

The total antenna efficiency $e_{0}$ is used to take into account losses at the input terminals and within the structure of the antenna. $e_{0}$ is due to the combination of number of efficiencies which is illustrated in the following equation (1):

$$
e_{0}=e_{r} e_{c} e_{d}
$$

Where $e_{0}=$ total efficiency, $e_{r}=$ reflection (mismatch), $\quad e_{c}=$ conduction efficiency, $e_{d}=$ dielectric efficiency.

The gain of the antenna is closely related to the directivity. In addition to the directional capabilities it accounts for the efficiency of the antenna. Gain arising from impedance mismatches (reflection losses) and polarization mismatches (losses). Gain is the ratio of the intensity, in a given direction, to the radiation intensity that would be obtained if the power accepted by the antenna were radiated isotropically. The gain is expressed by the following equation (2):

$$
\begin{gathered}
\text { Gain }=4 \pi \frac{\text { radiation int ensity }}{\text { total input accepted power }} \\
=4 \pi \frac{U(\theta, \phi)}{P_{i n}}
\end{gathered}
$$

We can write that the total radiated power $\left(P_{\text {rad }}\right)$ is related to the total input power $\left(P_{i n}\right)$ by (3) and (4)

$$
\begin{aligned}
P_{r a d} & =e_{c d} P_{i n} \\
G_{0} & =e_{c d} D_{0}
\end{aligned}
$$

The maximum value of the gain is related to the maximum directivity. The bandwidth is usually expressed as the ratio of the upper-to-lower frequencies of acceptable operation. For example, a 10:1 bandwidth indicates that the upper frequency is 10 times greater than the lower. The directivity of an antenna is defined as the ratio of the radiation intensity in a given direction from the antenna to the radiation intensity averaged over all directions. The average radiation intensity is equal to the total power radiated by the antenna divided by $4 \pi$. In mathematical form, it can be written as (5)

$$
D=\frac{U}{U_{0}}=\frac{4 \pi U}{P_{r a d}}
$$

Where $D=$ directivity, $U=$ power density, $U_{0}=$ radiation intensity of isotropic source, $P_{\text {rad }}=$ total radiated power.

\subsubsection{Fitness function evaluation}

The fitness function should be sufficiently fast to compute. It must quantitatively measure how fit a given solution is or how fit individuals can be produced from the given solution. Calculating the fitness function directly might not be possible due to the inherent complexities of the problem at hand. In such cases, we do fitness approximation to suit our needs. Fitness Function is helpful in chromosome evaluation which is a Genetic Algorithm part. The problem is to find a suitable Fitness Function for a chromosome evaluation to get a solution for Intrusion Detection. Fitness function is given by the formula (6) 


$$
F=\frac{a}{A}=\frac{b}{B}
$$

Where $A$ is Total of attacks, $a$ denotes Number of attack connections in which, the individual correctly classified. $B$ is Normal connections in the population. $b$ means number of normal connections through a network correctly classified. Fitness Function is working properly and effectively to help in reasonable evaluation.

The antenna having various parts called "lobes" which is classified as main lobe, back lobe, minor lobe and side lobe. In the antenna, side lobe level is the main problem which causes the wastage of energy at transmitter side. A side lobe is a radiation lobe in any direction other than the intended lobe. Usually it is adjacent to the main lobe. The ratio of power density in the lobe to the main lobe is called side lobe ratio or side lobe level (SLL).

The use of GA to optimize the spacing between elements, it is also utilized to get the optimum and achieves the minimum SLL. The fitness function, denoted by $f$, is merely chosen to be the side lobe level that is computed in the side lobe region as in (7)

$$
f=S L L=\max [20 \log (A F(\theta))]
$$

This is evaluated in the side lobe region that includes all lobes different from the main lobe. The symmetry dictates that the optimization should be done on half the array (the right-hand side one in this work) with the other half constructed by symmetry which is expressed in (8).

$$
A F(\theta)=\sum_{k=1}^{N} \cos [2 \pi x k(\cos \theta-\cos \theta 0)]
$$

In antennas are generally used for the application which require high gain and high directivity. To reduce side lobes level is to increase the beam width and reduced maximum power. We can reduce SLL for microstrip patch arrays up to $-45 \mathrm{db}$ level which is a very good achievement in reduction of SLL for better use in engineering. We studied different side lobe level reduction algorithm in antenna array such as particle swarm optimization (PSO), Ant colony algorithm (ACO), Invasive weed optimization (IWO) and spider monkey optimization (SMO). The Hybrid adaptive CSO combined with GA is more proficient than standard genetic algorithm. Compared to other methods such as genetic algorithms and simulated annealing, the improved cat Swarm optimization with genetic algorithm is much better performance in terms of the side lobe level and requires the least of mathematical pre-processing .

The three objectives considered are the minimization of the return loss, together with the minimization of the cross polarization and the mutual coupling between the ports. The optimization variables are directly linked to the antenna parameters and the analysis results are extracted and linked to the optimization targets. The optimization can always be manually stopped, if no further improvements are expected. Multi-objective optimization helps in understanding the physics of the problem while exploring the design space, looking for the set of optimal configurations. Antenna optimization aims at creating advanced and complex electromagnetic devices that must be competitive in terms of performance, serviceability, and cost effectiveness. An antenna design usually involves specifications related to directivity, impedance matching, crosspolarization, and frequency range, which can be formulated as both objective and constraint functions. Reducing search space not only speeds up the optimization but also potentially leads to problems with global optimality guarantees of solution. Multi objective design of antennas is an important and active field of inquiry.

\subsection{Cat Swarm based Genetic Optimization design}

A Cat swarm based genetic optimization approach for fast multi-objective optimization of multi-parameter antenna structures. The initial parameter in cat swarm optimization is seeking mode, tracing mode. These two modes are mathematically modelled to solve different optimization problems. The figure 2 shows seeking mode parameters of Cat swarm based Genetic Optimization design.

The Seeking Memory Pool (SMP) identifies the size of seeking memory for each cat also they indicate the points required by the cat. Seeking range of the selected dimension (SRD) is used to declare the mutative ratio for the selected dimensions. Self position considering (SPC) is a Boolean variable, which decides whether the point where the cat is already standing will be one of the candidates to move. Counts of dimension to change (CDC) are enclosing how many dimensions will be varied. 


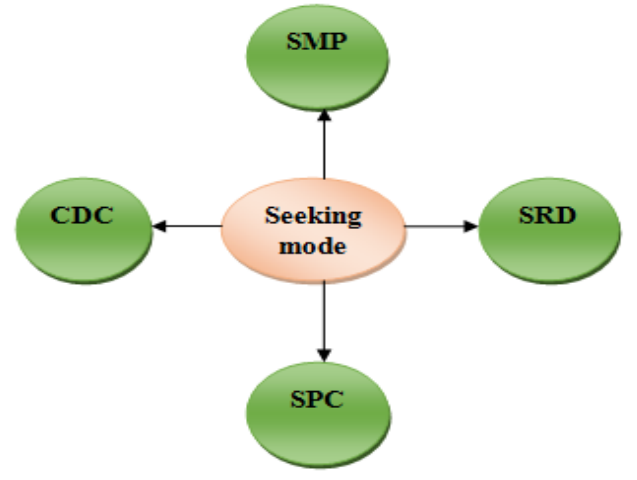

Figure 2: Seeking mode parameter

The steps involved in the seeking mode are:

Step 1: Make $j$ copies of the present position of cat $k$, where $j=S M P$. If the value of SPC is true, let $j=(S M P-1)$, then retain the present position as one of the candidates.

Step 2: For each copy, according to CDC, select the dimension as the candidates for changing.

Step 3: For the dimensions, which are selected in step 2 , in each copy randomly plus or minus SRD percents of the present values and replace the old ones.

Step 4: Calculate the fitness values $(F S)$ of all candidate points.

Step 5: If all $F S$ are not exactly equal. Calculate the selecting probability of each candidate point by equation (7), otherwise set all the selecting probability of each candidate point be 1 .

Step 6: Randomly pick out the point and its move to the candidate points and replace the position

$$
P_{i}=\frac{\left|F S_{i}-F S_{b}\right|}{F S_{\max }-F S_{\min }}, 0<i<j
$$

To find the minimum solution of the cost function is $F S_{b}=F S_{\text {max }}$ otherwise $F S_{b}=F S_{\text {min }}$. Where $F S_{\max }$ and $F S_{\min }$ are maximum and minimum cost functions.

The tracing mode parameters are random variable $\left(r_{1}\right)$, constant $\left(c_{1}\right)$. The random variable belongs to $[0,1]$ and the constant which is set to 2 in the experiments. The Tracing mode process of CSO is shown in below figure 3. The steps involved in this mode are as follows:

Step 1: Update the velocities for every dimension $(v)$ according to equation (8).

Step 2: Check if the velocities are in the range of maximum velocity. In case the new velocity is over range, set it be equal to the limit.

Step 3: Update the position of cat $k$ according to equation (9)

$$
\begin{gathered}
\boldsymbol{v}_{k, d}=w \cdot v_{k, d}+r_{j} c_{j}\left(x_{g b e s t, d}-x_{k, d}\right), d=1,2,3 \ldots M \\
x_{k, d}=x_{k, d}+v_{k, d}
\end{gathered}
$$

Where $v_{k, d}$ is velocity, $w \cdot v_{k, d}$ denotes Inertia weight ,$r_{j} c_{j}$ denotes Acceleration coefficient, $x_{\text {gbest }, d}$ is best position and $x_{d}$ denotes position.

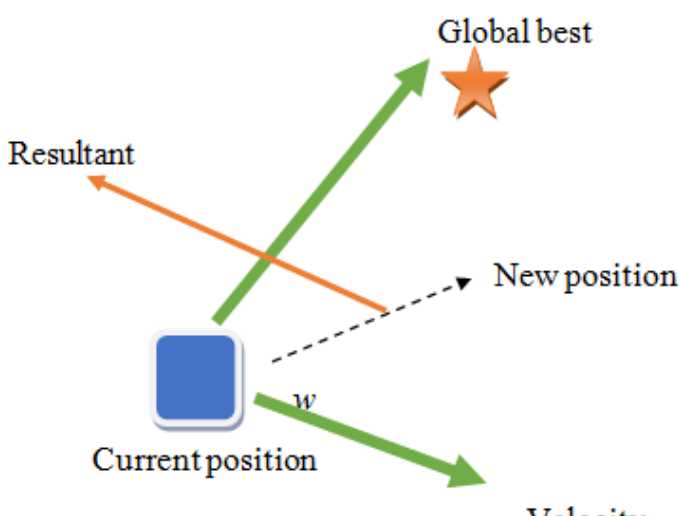

Figure 3: Tracing mode process

The CSO have different strategies for global and local searches. The update of velocity and position is used only for the global search cats in tracing mode, not for the local search cats in seeking mode. For the cats in seeking mode, their step size is selected by the SRD value chosen, not affected by the velocity and position. Without the large change in the velocity and position of seeking mode cats, they are possible to focus on an exhaustive search for the local area. Randomly picked dimensions to be mutated based on the CDC value provide a diverse movement of local cats for a better solution [23]. The SMP also provides a proper number of copies of a cat with regard to convergence speed. The searching cats are classified 
into these two modes. Each cat has its own position to be decided by a fitness function, velocity for each dimension, and a fitness value evaluated by a fitness function in the solution space. The solution space has a certain number of dimensions. Also, every cat has its flag indicating it is in either seeking mode or tracing mode. The number of seeking flags and tracing flags is decided by mixture ratio (MR). MR defines the ratio of the number of tracing mode cats to the number of seeking mode cats. Seeking node and tracing mode is also known as resting phase and movement phase. To improve the fitness function was finding the solution and reaches a better coverage; result in a better guidance of the search and thus in optimizations with less iteration.

\subsection{Efficient Genetic Optimization algorithm}

A Genetic Algorithm has significant advantages over traditional optimization techniques as follows. It can be applied in any problem and conducts a universal research in the solutions spaces. The algorithm does not require prior knowledge of the optimization problem. It does not depend on the initial conditions of the search. They used two optimize parameters such as continuous or discrete and GA works well with large number of variables. It can be run in parallel on multiple computers and optimizes variables with quite complex cost surfaces. The genetic algorithm is a best evolutionary mechanism. The following steps to be executed they are : (1) Fitness function or objective function it is calculated in individual.(2) Selecting the individuals are chosen from the current population as parents to be involved in recombination.(3) Recombination spring is formed from the parents by applying genetic operators are such as crossover and mutation. (4) Replacement: Some of the spring is replaced with some individuals. Genetic Algorithm contains sequence of operations, which are: Selection, Crossover, Mutation and sometimes Replacement, but the first operation is depending on the fitness value that obtained by Fitness Function.

GAs are simple and practical algorithm and easy to be implemented in antenna structures. The genetic optimization have reproduction is a process where the individual is selected to move to a new generation according to its fitness. The biased roulette wheel selection is employed. The probability of an individual's reproduction is proportional to its part on the biased roulette wheel. The main objective of crossover is to reorganize the information of two different individuals and to produce a new one. A single point crossover is applied and probability of crossover is selected as 1.0.To add a random integer from the range $[-10,10]$. Mutation is used to introduce some sort of artificial diversification in the population to avoid premature convergence to local optimum. The above-mentioned operations of selection, crossover and mutation are repeated until the best individual is found. Mutation helps to maintain diversity in population.

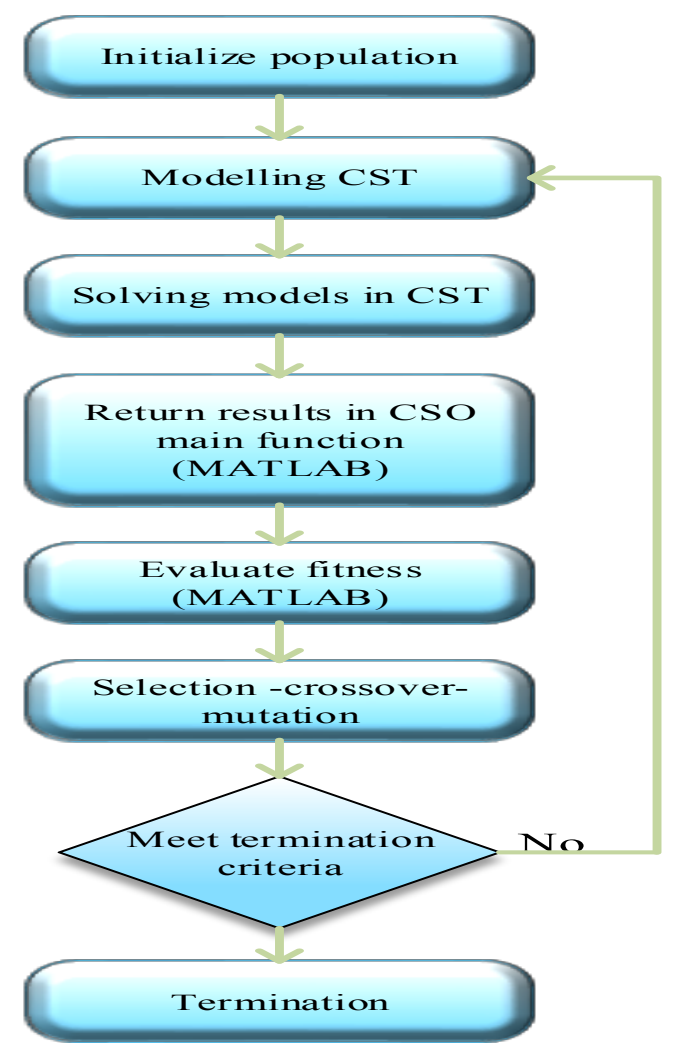

Figure 4: Proposed flow diagram for Genetic Algorithm

There are two objectives in genetic algorithm. The first objective is high gain antenna with low side lobes and second one is different planes. Use a multiobjective genetic algorithm (MOGA) in place of the cat swarm based genetic algorithm. MOGA are wellsuited for optimization problems in antenna. The genetic algorithm is a robust, stochastic search method that models its processes after the principles of natural selection and evolution [24]. These genetic algorithms are very useful for finding optimum antenna designs that maximize or minimize certain radiation properties. The GA provides optimal solutions by successively creating populations that improve over many generations. The above figure 4 illustrates the proposed flow chart for Genetic Algorithm. 
Step 1: Create initial population by generating a random string of binary numbers.

Step 2: Create models according to the genes of individuals in population, set solving conditions and solve models in CST. Then return calculated $S_{11}$ parameters to main function automatically (Matlab). The returned parameter $S_{11}$ will be called by fitness function (Matlab).

Step 3: Evaluate the fitness function. If the stopping criteria are satisfied then stop, else go to2.

Step 4: The fitness function is the link between the physical problem and optimization procedure. The appropriate solution depends on the mathematical formulation of this function. Several researchers use the relationship Equation (10) to express the cost function.

$$
\begin{gathered}
\operatorname{Cos} t=\frac{1}{N} \sum_{i=1}^{N} Q\left(f_{i}\right) \\
Q\left(f_{i}\right)=\left\{\begin{array}{l}
\left|S_{11}\left(f_{i}\right)\right| \text { for } S_{11} \geq-10 d B \\
+10 d B \text { for } S_{11} \leq-10 d B
\end{array}\right.
\end{gathered}
$$

We improve the cost function according to the Equation (10). The fitness function is defined as in (12)

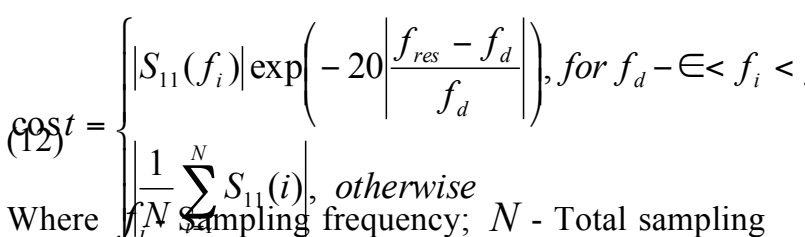
points, $f_{d}$-Desired frequency; $f_{\text {res }}$ - The individual resonance frequency; $S_{11}$-The $S_{11}(d B)$ parameter at resonance frequency. $=0.1$.

\section{Simulation Results and Discussion}

If minimum of return loss parameter is on left side of resonant frequency Keep Yo fixed and reduces the length of patch in step size then this minimum will shift towards resonant frequency and we can obtain the minimum of return loss parameter at specified resonant frequency and can be seen from figure 5 .

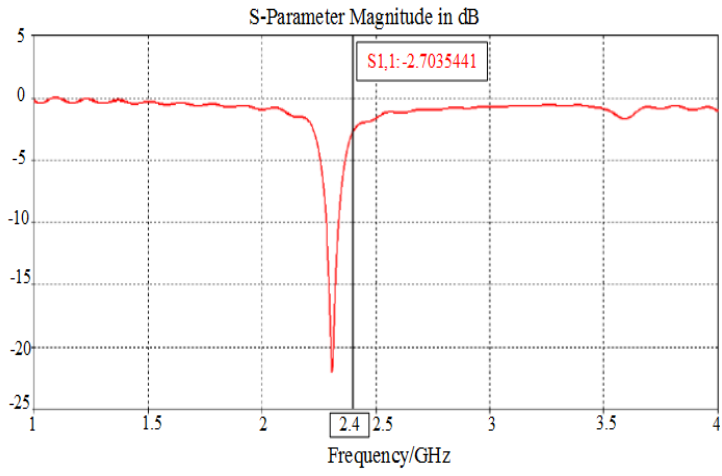

Figure 5: Return loss parameter for resonating antenna

The two algorithms produce different sets of solution values. The radiation pattern obtained from the CSO and CSGO algorithm are shown in Figure 6. It can be observed that the results obtained from CSO satisfy the design criterion very well. The results from CSGO maintain the desired pattern for the most part, with only a few side lobes exceeding the limit. This implies that CSGO has not converged perfectly. This fact can be further illustrated by observing the fitness function values. Most antenna arrays are designed to produce a directive beam at a particular direction and while keeping the side lobe level (SLL) small to avoid interference with other radiating sources.

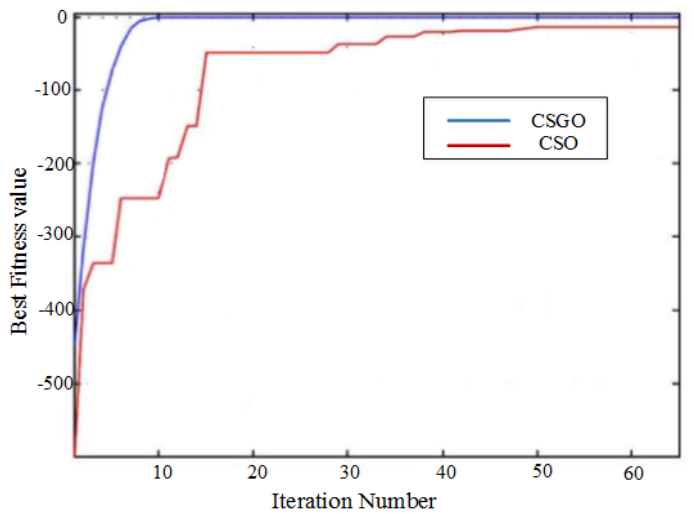

Figure 6: Fitness value versus iteration number for CSGO and CSO algorithm for the first design problem.

The optimized antenna resonates at three frequencies (3.5 GHz, $4.76 \mathrm{GHz}$ and $5.62 \mathrm{GHz}$ ) as shown in figure 7 . The slots are responsible for obtaining the perfect impedance matching in the bands. The reflection coefficient is improved from $-25.65 \mathrm{~dB}$ to $-32.66 \mathrm{~dB}$. It can be seen that for CSGO, the fitness 
function value reaches its maximum possible value zero within 20 iterations. The saturation of fitness value implies convergence.

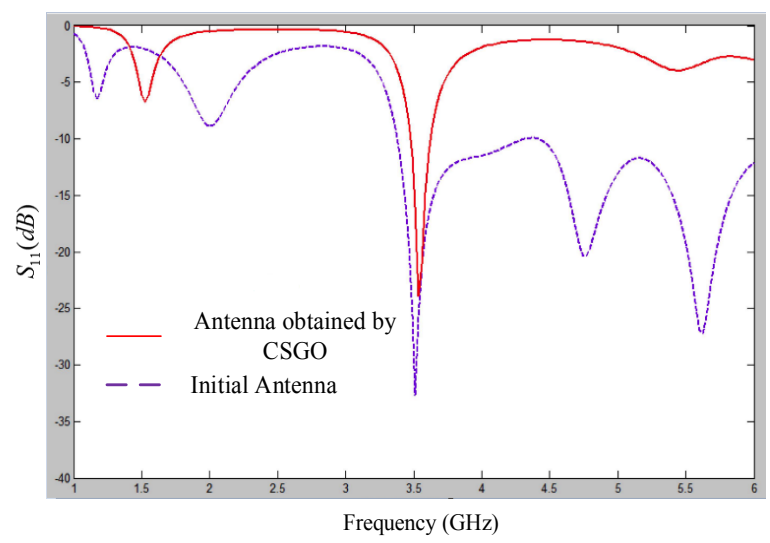

\section{Figure 7: Simulated initial antenna and obtained antenna by CSGO}

CSO had a side lobe level of about $-22 \mathrm{dBm}$. CSGO has a side lobe level of about $-24 \mathrm{dBm}$ and genetic optimization has the least SLL of about $-30 \mathrm{dBm}$. In general, all the optimization methods reduced the SLL to some extent, but they have different behaviour at different frequencies. Both results are obtained using the same fluid simulation. The result shows that the required simulation time of the proposed algorithm is greatly reduced with a similar accuracy which is shown in below figure 8 .

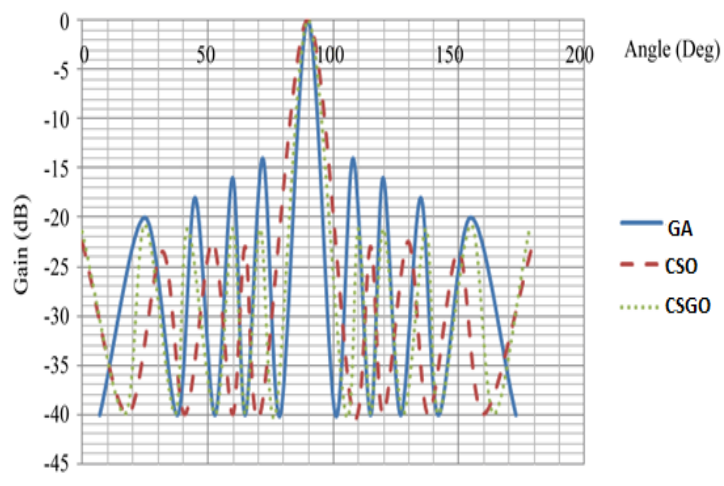

Figure 8: Side love level reduction in CSGO

The total average power consumption along the signal path can be divided into two main components: the power consumption of all the power amplifiers $P_{P A} \quad$ and the power consumption of all other circuit blocks $P_{c}$. The first term $P_{P A}$ is dependent on the transmit power $P_{\text {out }}$, which can be calculated according to the link budget relationship .In order to consider the total energy consumption, all signal processing blocks at the transmitter and the receiver need to be included in the model. However, in order to keep the model from being over-complicated at this stage, baseband signal processing blocks are source coding, pulse-shaping, and digital modulation.

The power consumption of the power amplifiers can be approximated by using (13)

$$
P_{P A}=(1+a) P_{\text {out }}
$$

The total energy consumption per bit for a fixed-rate system can be obtained by the formula (14)

$$
E=\left(P_{p A}+P_{c}\right) / R_{b}
$$

Energy-efficient communication techniques typically focus on minimizing the transmission energy only, which is reasonable in long-range applications where the transmission energy is dominant in the total energy consumption.

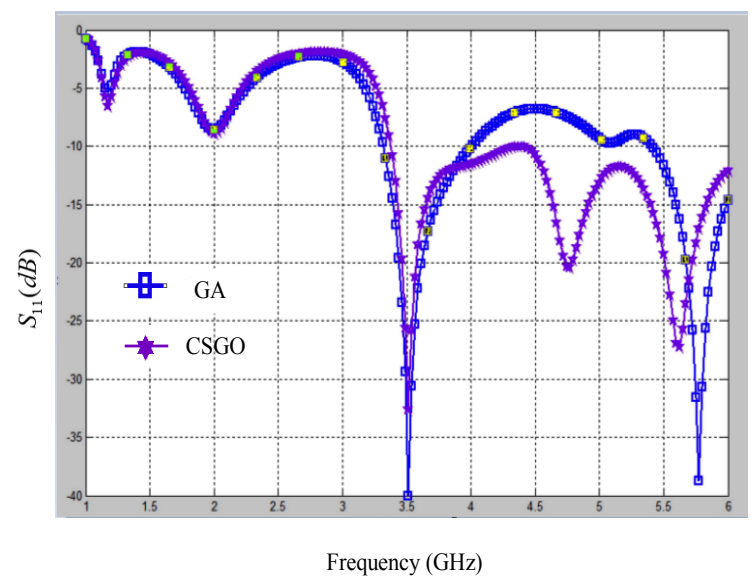

Figure 9: Optimized antenna obtained by GA and CSGO

The use of gridded ground plane with overlapping cells is proposed in this paper, GA and CSGO find the best ground plane geometry with improved performance more effectively. Comparisons of the reflection coefficient over frequency in the optimized designs are shown in Figure 9. We can easily visualize from these plots that a significant improvement in bandwidth has been achieved with the proposed new ground plane for the low-frequency band as well as for the high-frequency band. CSGO and GA give similar geometry and show correlation of $82.34 \%$.

\section{Conclusion}

This paper proposed a hybrid method of antenna optimization using CSGO algorithm. We propose a new optimization algorithm which adjusts the 
optimization efficiency of each service in order to reduce the simulation complexity. In results, we show that the proposed algorithm performs faster and more effectively than the previous algorithm. The cat swarm based genetic algorithm optimization of the parameters of an antenna and performance analysis using the CST software has been carried out the interdisciplinary convention. The paper makes three main contributions: (i) In almost all design configurations, the null depth improves by approximately $-80 \mathrm{~dB}$. (ii) The maximum SLL is also reduced in all cases. The separations between the array elements are determined using firefly algorithm and PSO algorithm. It has been found that the design obtained from FA satisfies the predefined SLL beam width and null requirements very well. Overall, the FA is found to be very suitable for NUSLA design compared to other existing optimization-algorithmbased methods. CSO systems are more energyefficient than CSGO systems and also obtain the better computational cost.

\section{References}

[1] Majumder, Alak. "Rectangular microstrip patch antenna using coaxial probe feeding technique to operate in S-band." International Journal of Engineering Trends and Technology (IJETT), Vol 4, No 4, 2013.

[2] Sethi, Sarinka, and Er Manish Mehta. "Microstrip Patch Antenna for UWB Applications: An Overview." International Journal of Engineering Science, PP 7501, 2016.

[3] Khan, Abdul Qadir, Muhammad Riaz, and Anas Bilal "Various Types of Antenna with Respect to Their Applications: A Review." International Journal of Multidisciplinary sciences and Engineering, Vol 7 No.3, 2016.

[4] Sarma, Angana, et al. ,"Soft-Computing-Based Optimization of Low Return Loss Multiband Microstrip Patch Antenna." Handbook of Research on Natural Computing for Optimization Problems. IGI Global, PP 583-617, 2016.

[5] Koyya, L., R. L. Valluri, and G. Raju. "Optimization of geometry of microstrip patch antenna for broadband applications." International Journal of Advance Research in Electrical, Electronics and Instrumentation Engineering, Vol 2, No 7. PP :3119-3123, 2013.

[6] Bhomia, Y., Prasad, S.V.A.V. and Kumar, P.,"Designing of Rectangular Fractal Microstrip Patch Antenna using Iteration Methods" European
Journal of Advances in Engineering and Technology, Vol 1 No 1. PP48-53, 2014

[7] Darshana R. Suryawanshi, Prof. Bharati A. Singh,A "Compact Rectangular Monopole Antenna with Enhanced Bandwidth", IOSR Journal of Electronics and Communication Engineering (IOSR-JECE), Vol 9, No 2, Ver. VII (Mar - Apr.), PP 54-57, 2014.

[8] Huang, Li, et al. "Impact of resonator geometry and its coupling with ground plane on ultrathin metamaterial perfect absorbers." Applied Physics Letters, Vol 101, No 10. PP 101-102, 2012.

[9] Rocca, Paolo, et al."Predicting antenna pattern degradations in microstrip reflectarrays through interval arithmetic." IET Microwaves, Antennas \& Propagation Vol 10, No.8. PP 817-826, 2016.

[10] Linden, Derek S. "Antenna design using genetic algorithms ."Proceedings of the 4th Annual Conference on Genetic and Evolutionary Computation. Morgan Kaufmann Publishers Inc. 2002

[11] Al-Azza, A. A., Al-Jodah, A. A., \& Harackiewicz, F. J. "Spider monkey optimization": A novel technique for antenna optimization, Vol 15, 1016-1019, 2016.

[12] Poian, M., et al. "Multi-objective optimization for antenna design." Microwaves, Communications, Antennas and Electronic Systems, 2008. COMCAS 2008. IEEE International Conference on. IEEE.

[13] Koziel, Slawomir, and Adrian Bekasiewicz.v "Low-cost multi-objective optimization of antennas using Pareto front exploration and response features." Antennas and Propagation (APSURSI), IEEE International Symposium on. IEEE. 2016.

[14] Koziel, Slawomir, and Stanislav Ogurtsov. "Antenna design by simulation-driven optimization." Springer Science \& Business Media, 2014.

[15] Koziel, Slawomir. "Objective Relaxation Algorithm for Reliable Simulation-Driven Size Reduction of Antenna Structures." IEEE Antennas and Wireless Propagation Letters. 2017.

[16] Gupta, Kriti Priya. "Genetic Algorithm Approach for Bandwidth Optimization in Near Video on Demand System." International Journal of Computer Science and Information Technologies, Vol. 5, No 6,.PP 7430-7438, 2014. 
[17] Travassos, X. L., D. A. G. Vieira, and A. C. Lisboa, "Antenna optimization using multiobjective algorithms." ISRN Communications and Networking, Vol 4, 2012.

[18] Modi, Anuj Y., Jigar Mehta, and Nilima Pisharody. "Synthesis of Elliptical Patch Microstrip Antenna using artificial neural network." In Microwave and Photonics (ICMAP), 2013 International Conference on, pp. 1-3. IEEE, 2013.

[19] Pujara, Dhaval, Anuj Modi, Nilima Pisharody, and Jigar Mehta. "Predicting the performance of pyramidal and corrugated horn antennas using ANFIS." IEEE Antennas and Wireless Propagation Letters Vol. 13, pp: 293-296, 2014.

[20] Kalambe, Nita, Dhruv Thakur, and Shubhankar Paul. "Review of Microstrip Patch Antenna Using UWB for Wireless Communication Devices." IJCSMC , Vol 4, No 1 PP 128-133, 2015.

[21] Pappula, Lakshman, and Debalina Ghosh. "Linear antenna array synthesis using cat swarm optimization." AEU-International Journal of Electronics and Communications, Vol 68, No 6. PP 540-549, 2014.

[22] Ram, Gopi, et al. "Cat swarm optimization as applied to time-modulated concentric circular antenna array: Analysis and comparison with other stochastic optimization methods." IEEE Transactions on Antennas and Propagation. Vol 63, No 9. PP 4180-4183, 2015.

[23] So, Jinhyun, and W. Kenneth Jenkins. "Comparison of cat swarm optimization with particle swarm optimization for IIR system identification." Signals, Systems and Computers, Asilomar Conference on. IEEE. 2013

[24]Lamsalli, Mohammed, et al."Genetic Algorithm Optimization for Microstrip Patch Antenna Miniaturization." Progress In Electromagnetics Research Letters Vol 60. PP 113-120, 2016. 